\title{
Teriomorfismo e malinconia \\ Una storia notturna della Sicilia: Nottetempo, casa per casa di Consolo
}

\section{Rossend Arqués}

Universitat Autònoma de Barcelona

\section{Abstract}

L'articolo vuol essere una lettura di Nottetempo, casa per casa che prende spunto dall'analisi del sostrato mitico e iconografico delle rappresentazioni teriomorfiche più importanti del romanzo (il «luponario»e il "caprone») per sottolineare il cammino del protagonista dall'afasia animale e malinconica in cui è immersa la sua famiglia, e lui stesso, all'accesso al linguaggio accompagnato dalla presa di coscienza della decadenza della società siciliana che coincide anche con il suo esilio.

Parda chiavi: Vincenzo Consolo, licantropia, Pan, malinconia, letteratura siciliana.

\section{Abstract}

The article aims at providing a reading of Nottetempo, casa per casa, starting with the analysis of the mythological and iconographic substrata of the most significant theriomorphic representations within the novel (the "lycanthrope» and "goat») in order to emphasise the path taken by the protagonist from that animal, melancholy aphasia in which he and his family are immersed, through to the access to language, accompanied by the growing awareness of the decadence of Sicilian society that also coincides with his exile.

Key words: Vincenzo Consolo, lychanthropy, Pan, melancholy, Sicilian literature.

\section{Nottetempo... all'interno del trittico consoliano}

La notte, la notte mitica, ha tutti gli elementi dell'incubo. Nottetempo, casa per casa $(1992)^{1}$ nella trilogia che ha inizio con $\mathrm{Il}$ sorriso dell'ignoto marinaio (1976) e si conclude con Lo spasimo di Palermo (1998), occupa il secondo momento del periodo storico che Consolo ha dedicato alla Sicilia, il primo essendo il Risorgimento e gli anni che precedono l'assassinio del giudice Bor-

1. Cit. dall'edizione Nottetempo, casa per casa, Introduzione di Antonio Franchini, Milano: Mondadori (Oscar Scrittori del Novecento), 1994 [1992]. 
sellino. ${ }^{2}$ Si tratta di un periodo della storia siciliana (siamo negli anni Venti), quello di Nottetempo, nero come le camicie dei fascisti che piano piano riempiono le piazze dei paesi e delle città d'Italia. Più concretamente, ci troviamo a Cefalú, già scenario de $I l$ sorriso... ${ }^{3}$ Anni difficili, non solo per le vicende politiche, ma anche e soprattutto per quelle morali, come tanti altri della storia italiana. Consolo ha sempre agito come una memoria attenta e sensibile del passato che viene accostato agli avvenimenti più recenti di cui egli è testimone e interprete. In Nottetempo... Consolo stabilisce implicitamente un nesso tra l'Italia degli anni Venti e quella degli anni Settanta, così come nel Sorriso... tra il Risorgimento e gli anni Sessanta e nello Spasimo... tra gli anni 30 e l'inizio degli anni 90, con le morti di Falcone e Borsellino. Fasi storiche tutte segnate da successive cadute della società isolana e continentale in un pozzo senza uscita e senza possibilità di riemersione. "Traversare la Sicilia intera scrive Consolo in un articolo intitolato «Paesaggio metafisico di una follia pietrificata $»^{4}$ - , visitare quelle città e quei paesi un tempo vitali per umanità e cultura, carichi dunque, ancora fino a pochi anni addietro, di volontà e di speranza, luoghi che il moderno feticcio dell'accelerazione spasmodica, l'autostrada, ha tagliato fuori dallo spazio (...); visitare oggi Enna, Caltanissetta (...) più che accenderti furori, inutili ormai, ti infonde sconsolazione e pena. Sono paesi che si sono svuotati d'uomini e significato». 5

Seguendo gli esempi di Manzoni, Verga e, soprattutto di Tomasi di Lampedusa e Sciascia, l'autore sceglie la formula narrativa del romanzo storico con questo obiettivo: collocare in figure, luoghi e trame del passato problemi e sentimenti del presente, che altrimenti risulterebbero non solo difficili da trattare ma addirittura pericolosi. Ne era ben conscio Calvino quando stabilì un parallelismo tra la narrazione realista e la Medusa. Per sfuggire allo sguardo pietrificante del mostro mitologico non resta nessun altro modo che osservare la realtà attraverso la corazza-specchio di Perseo. La narrazione storica di Consolo ha questa funzione di corazza-specchio: proprio allontanandosi dal presente, essa è in grado di coglierlo, analizzarlo e giudicarlo, mentre riesce contemporaneamente a immergersi con spirito critico e analitico nel momento storico preso di volta in volta in esame, senza mai tralasciare la dimensione del mito nelle sue diverse sfaccettature, soprattutto di quei miti arcaici che hanno dato essenza e forma alla natura umana e morfologica della terra di Sici-

2. Come lo stesso Consolo ha dichiarato in «Le interviste d'Italialibri» 2001, anche se molte altre sue opere percorrono episodi ed eventi dell'isola: quello greco (Le pietre di Pantalica), quello dominato dagli spagnoli (Lunaria), quello illuministico (Retablo), ecc.

3. «E Cefalù è stata un approdo, un luogo d'elezione e di passione. Perché Cefalù e non Palermo? Cefalù perché microcosmo, antifona di quel gran mondo che è Palermo [...]. Io ho scelto Cafalù. Sono piccoli mondi così ricchi dentro il cui viaggio, la scoperta può non finire mai.» «La corona e le armi», Giornale di Sicilia, 14 marzo 1981.

4. Pubblicato il 19 ottobre 1977 su Il Corriere della sera, cit. da Francesco GIOVIALE, «L'isola senza licantropi», in Id., Scrivere la Sicilia. Vittorini e oltre, Siracusa: Ediprint, 1983, p. 165-176.

5. Lo stesso messaggio possiamo trovarlo in altri articoli e testi suoi. Citiamo a mo' di esempio «La cultura siciliana è tramontata per sempre», L'Ora, 31 maggio $1982 \mathrm{e}$ «Flauti di reggia o fischi di treno?», Il Messaggero, 19 luglio 1982. 
lia. Una narrazione storica, cioè, rivolta programmaticamente a evitare ogni realismo politico e storico, e alla ricerca di un nuova lingua letteraria capace di unire, condensare e quindi conservare fuse tra loro tutte quelle tracce. Di questo aveva già parlato Giovanardi in una sua bellissima recensione a Nottetempo... in cui sostiene che piú che a una storia questo romanzo sembra dar vita a una contrapposizione tra due diversi modelli di vita e di cultura, due modelli antropologici da sempre compresenti nello spazio insulare, ${ }^{6}$ e cioè la cinica accettazione dell'esistente e il pervicace ricorso alla fantasia come fuga dalla realtà. ${ }^{7}$ Ciò detto, però, non si può dimenticare che lo stesso Consolo ha insistito piú volte sulle fratture prodottesi nel tessuto sociale, ambientale e antropologico insulare in periodi concreti della storia siciliana piú recente (si veda a questo proposito il citato articolo Paesaggio metafisico... in un punto del quale leggiamo: «È il paesaggio della nuova umanità siciliana, uguale ormai, per perdita di cultura e di identità, alle altre realtà regionali italiane nate dallo squallore consumistico degli anni del cosiddetto boom economico»). ${ }^{8}$

Per stabilire fin dall'inizio la metodologia di questo mio lavoro, devo informare che la mia lettura di Nottetempo... parte dalle teorie analitiche junghiane e, in particolar modo, di quelle di Hillman, non perché io abbia una particolare predilezione per questo approccio critico, ma perché credo che effettivamente questo testo narrativo, come gran parte dell'opera consoliana, trovi spunto nell'universo degli archetipi di derivazione junghiana. ${ }^{?}$

6. Stefano GIOVANARDI, "Dalla follia alla scrittura», in La Repubblica, 25 aprile 1992 [ora in Nuove effemeridi, n. 29, 1995, p. 152]. Si vedano anche Giulio FERRONI, «Bestie trionfanti», in L'Unità, 27 aprile 1992 [ora in Nuove effemeridi, n. 29, 1995, p. 153-157]; Francesco Gioviale, "L'isola senza licantropi», in Id., Scrivere la Sicilia. Vittorini e oltre, Siracusa: Ediprint, 1983, p. 168; Francesco GIOvIALE, op. cit., p. 168; Lorenzo Mondo, «Invasati e dolenti", in La Stampa, 4 aprile 1992 [ora in Nuove effemeridi, n. 29, 1995, p. 147-148]; Attilio SCUDERI, Scrittura senza fine. Le metafore malinconiche di Vincenzo Consolo, Enna: Il Lunario, 1997; Giuseppe Traina, Vincenzo Consolo, Fiesole: Cadmo, 2001.

7. L'autore stesso in un articolo del 1986 intitolato «Sirene siciliane» (ora in Vincenzo CoNSOLO, Di qua dal faro, Milano, Mondadori, 2001, p. 175-181), tenta di dividere la letteratura siciliana in due grandi rami: "occidentale e orientale, storico e esistenziale, poetico-lirico e prosaico, mitico e razionale, simbolico e metaforico", con tutti gli spostamenti e le contraddizioni del caso, fino ad ipotizzare una letteratura siciliana che riesca a fondere i due mondi: «E poi a me sembra di aver capito che tu, come capita ad alcuni siciliani della specie migliore, sei riuscito a compiere la sintesi di sensi e di ragione - dice La Ciura a Corbera» [i due personaggi del racconto Lighea di Tommasi di Lampedusa]. Quella sintesi che lui stesso tenterà in Nottetempo..., se non in tutta la sua opera.

8. Un argomento simile si legge nell'articolo di Vincezo CONSOLO, «La cultura siciliana è tramontata per sempre», pubblicato su L'Ora (31 maggio 1982): "Con la seconda rivoluzione industriale, con la rivoluzione tecnologica, con i movimenti umani avvenuti nel nostro Paese in questi ultimi trent'anni, con i cambiamenti antropologici che questi movimenti hanno comportato, sono finite le culture locali, quelle che avevano una loro precisa individualità, una loro storia, una loro realtà «realtà». La cultura siciliana secondo me è tramontata».

9. L'influsso hillmaniano è accertato. Lo stesso Consolo mi ha informato che un suo amico, Giovanni Reale, analista e filosofo junghiano, l'aveva introdotto a questo tipo di letture, tra cui c'erano anche alcuni libri di HILlMAN di cui qui mi preme sottolineare in particolar modo, Saggio su Pan, Animali del sogno e Il sogno e il mondo infero. In un articolo intitola- 


\section{Licantropia: dolore e cosciencia}

Di che notte si tratta e quali sono le creature che vivono quella pittura notturna dominata dalla animalità e dal teriomorfismo? Passiamo ad analizzare, dunque, dettagliatamente e in profondità le diverse facce della bestialità e le funzioni che esse hanno nel testo oggetto della nostra attenzione. Non avrebbe senso, infatti, ridurre a una sola tutte le forme di animalità presenti nell'opera, omettendo di mettere in luce le differenze morfologiche e mitologiche di ciascuna di loro. ${ }^{10}$

Il romanzo ha inizio con la descrizione di un notturno lunare dominato da un uomo in pieno delirio, il cui terribile ululato squarcia il silenzio delle tenebre. Dietro a lui vaga attenta e angosciata un'altra figura, che in seguito sapremo essere il figlio, vero protagonista del romanzo. Il licantropo è Marano (il cognome derivato dallo spagnolo marrano ci dice delle sue origini di ebreo convertito a forza), padre di Pietro, vedovo. Il suo dolore straziante, insieme a quello di tutta la sua famiglia, è causato sia dal lutto per la perdita della sposa, sia dalla "memoria genetica» della violenza subita dagli antenati ebrei obbligati a cambiare cultura e religione, ${ }^{11}$ sia dall'ascesa sociale che lui e la sua famiglia hanno recentemente sperimentato grazie all'eredità lasciatagli da un eccentrico possidente locale, una specie di libertario tolstoiano che ha voluto beneficare la famiglia Marano al posto del suo legittimo erede, il nipote imbecille. Questo cambio repentino e non voluto di classe sociale sarà responsabile di una specie di schizofrenia comportamentale, almeno per quei personaggi femminili che si sentono obbligati a osservare scrupolosamente le regole sociali della piccola borghesia, per quanto diverse da quelle in cui sono nati e cresciuti. ${ }^{12}$ Un chiaro esempio di questo ci è offerto dal rifiuto di Lucia non solo di accettare ma neppure di ascoltare la dichiarazione d'amore di Janu, il capraio e amico d'infanzia, ora considerato solo un puzzolente pezzente.

La famiglia Marano corrisponde a un catalogo di figure malinconiche, a una "follia dai molti volti», come scrive Segre. ${ }^{13}$ Serafina, la sorella maggiore di Pietro, rappresenta l'immagine stessa della fenomenologia depressiva che la porta all'immobilità corporale, alla pietrificazione autistica o alla schizo-

to «Sirene siciliane» (ora in Vincenzo CONSOLO, Di qua dal faro, cit., p. 175-181) che prende spunto dal racconto Lighea, di Tomasi di Lampedusa, per parlare di altre sirene siciliane, tra cui Giovanni Reale, leggiamo (p. 181): «Se è vero, come sostiene Hilmann, che la pratica analitica non è altro che poiesis, quella di Reale lo è pienamente».

10. Come fa, invece, Rosalba GalVAGno, «Destino di una metamorfosi nel romanzo Nottetempo, casa per casa», in Enzo PAPA (a cura di), Per Vincenzo Consolo, San Cesario di Lecce: Manni, 2004, p. 23-58, che considera l'intero testo di Nottetempo... solo dal punto di vista della licantropia, senza avvertire la dialettica tra questa forma di animalità e le altre forme di trasformazione umana in bestia che sono presenti nello stesso testo consoliano.

11. «Da quel cognome suo forse di rinnegato, di marrano di Spagna o di Sicilia, che significava eredità di ànsime, malinconie, rimorsi dentro le vene» - si legge a p. 42.

12. Come chiarisce Vincenzo CONSOLO in «Le interviste d'Italialibri» 2001.

13. Cesare SEGRE, "Una provvisoria catarsi», in Corriere della sera, 19 aprile 1992 [ora in Nuove effemeridi, n. 29, 1995, p. 150]. 
frenia catatonica, ${ }^{14}$ così come ci è stata rappresentata iconograficamente nei secoli. ${ }^{15}$ La figlia minore, Lucia, invece, psichicamente alterata dal nuovo status della sua famiglia, è affetta da una psicosi maniaco-depressiva, in preda, com'è, a un continuo delirio paranoico. ${ }^{16}$ Parleremo di Pietro in seguito, dedicandogli più ampio spazio, per adesso occupiamoci del padre e del suo male «siciliano", come lo definisce Cervantes nel Persiles $(\mathrm{I}, 18) .{ }^{17}$ Non c'è dubbio che la descrizione cervantina, lasciando da parte il particolare del movimento in gruppo ("en manada») dei lupi, è presente sia nell'immaginario narrativo di Mal di luna di Pirandello ${ }^{18}$ che nel testo di Consolo, pur con la presenza in entrambi dell'elemento lunare, ${ }^{19}$ assente, invece, in Cervantes. Colpisce,

14. «E Serafina, ch'aveva preso il ruolo della madre e poi s'era seduta, fatta muta ogni giorno, immobile, di pietra, dentro nella scranna, il solo movimento delle dita che sgranano il rosario di poste innumerevoli, di meccaniche preghiere senza soste.» - si legge a 42. Per una tassonomia attuale di questa malattia depressiva si veda Eugenio BorgnA, Malinconia, Milano: Feltrinelli, 1992 e Marie-Claude LAмвотте, Esthétique de la mélancolie, Paris: Aubier, 1984. Quest'ultima vede nell'inibizione il tratto più significativo della malinconia moderna.

15. Ricordiamo qui le incisioni del Maestro F. B. (Franz Brun?) (1560), A. Janssens (1623), D. Feti (1614) o C. Friedrich (1818), si veda Raymond KLIBANSKY, Erwin PANOFSKY, Fritz SAXL, Saturno e la malinconia, Torino: Einaudi, 1983.

16. Il narratore ci parla della sua malattia a p. 45-48, da dove prendiamo le seguenti frasi: «Lei era innocente, immacolata come la bella madre. E stava ore e ore chiusa nella stanza, avanti alla toletta a pettinarsi» (...) Finché un giorno, un mezzogiorno (...) non si mise a urlare disperata sul balcone, a dire che dappertutto, dietro gli ulivi le rocce il muro la torre le sipale, c'eran uomini nascosti che volevano rapirla, farla perdere, rovinare (...) «Mi parlano, mi parlano!» diceva stringendo la testa fra le mani». «Nell'orecchio, nel cervello, vigliacchi!' e torbidi erano i suoi occhi, più neri nel pallore della faccia.». Per una tassonomia moderna della sua delirante afezione psicotico-paranoica si veda Eugenio BorgNA, Op. cit.

17. «Lo que se ha de entender desto de convertirse en lobos es que hay una enfermedad, a quien los médicos llaman manía lupina, que es de calidad que, al que la padece, le parece que se haya convertido en lobo, y se junta con otros heridos del mismo mal, y andan en manadas por los campos y por los montes, ladrando ya como perros o ya aullando como lobos; despedazan los árboles, matan a quien encuentran y comen la carne cruda de los muertos, y hoy día sé yo que hay en la isla de Sicilia (que es la mayor del Mediterráneo) gentes deste género, a quienes los sicilianos laman lobos menar, los cuales, antes que les dé tan pestífera enfermedad lo sienten y dicen a los que están junto a ellos que se aparten y huyan dellos, o que los aten o encierren, porque si no se guardan, los hacen pedazos a bocados y los desmenuzan, si pueden, con las uñas, dando terribles y espantosos ladridos.», Miguel de Cervantes, Los trabajos de Persiles y Sigismunda, ed. di Carlos Romero, Madrid: Cátedra, 2002, p. 244. D’altronde l'autore del Quijote poco si allontana dalla iconografia tradizionale dell'uomo lupo, così come è attestata nel Licaone di Pausania o di Ovidio. Si veda Paola MicozzI, "Tradición literaria y creencia popular: el tema del licántropo en Los trabajos de Persiles y Segismunda de Cervantes», Quaderni di filologia e lingue romanze, III serie, n. 6, 1991, p. 107-152.

18. Luigi PIRANDELlo, "Mal di luna», in Novelle per un anno, vol. II, t. I, a cura di M. Costanzo, Introduzione di G. Macchia, Milano: Mondadori, 1987, p. 486-495.

19. La presenza della luna, invece, diventa elemento costante e ricorrente in particolar modo a partire dal XIII secolo, anche se nel Satyricon di Petronio era già stata descritta una metamorfosi lupina al chiaro di luna. Si vedano Gaël Milin, Le chiens de Dieu, Centre de Recherche Bretonne et Celtique, 1993 (trad. it. El licantropo, un superuomo?, a cura di Jose Vincenzo Molle, Genova: ELCIG, 1997: p. 60-64) e P. MEnARD, Les lais de Marie de France, París: P.U.F., 1979. In quest'ultimo leggiamo a p. 222-223. "So bene — scrive Gervasio di Tilbury al capitolo CXX (De hominibus qui fuerunt lupi) della terza parte degli Otia - che nel 
in merito a questo, la supplica che il malato dirige ai familiari di allontanarsi da lui quando arriverà l'attacco del male. Inoltre è importante qui sottolineare che il male della licantropia ${ }^{20}$ viene attribuito da Cervantes alla terra siciliana, ${ }^{21}$ e abbiamo già visto che Consolo nel citato articolo Paesaggio metafisico... dichiara che l'identità arcaica dell'isola risiede appunto nell'esistenza di forme di bestialità terrorifica:

nostro paese càpita ogni giorno che certe persone siano mutate in lupo sotto l'influsso delle lunazioni (per lunationes mutantur in lupus)». Con il corpo coperto di pelo, l'infermo correva per i boschi, in preda al delirio, celandosi allo sguardo altrui e attaccando ferocemente persone o animali con cui s'imbatteva. Passata che era la crisi, la persona malridotta, di solito un uomo (è una malattia prettamente maschile, per quanto ci siano versione femminili di trasformazioni zoomorfe, come la donna-pantera), tornava in sé dimentico dell'attacco di follia. Questa versione del problema ha alimentato molta letteratura scritta e cinematografica, fino al punto che Boris Vian (Le loup-garou) ne fece una versione inversa, il lupo che si trasforma in uomo, cioè, il lupo-uomo.

20. Chiamati anche ulfhednar (uomini vestiti di pelli di lupo), i lupi mannari facevano parte tranquillamente delle truppe delle antiche popolazioni germaniche e scandinave in quanto eccezionali combattenti. La licantropia (dal greco lykos, lupo, e anthropos, uomo), ciò̀ la trasformazione di un uomo in «lupo mannaro», il «luponario», per effetto di una forza malvagia o di uno spirito animale capace d'impossessarsi di un essere umano e agire attraverso di lui, non solo ha riempito leggende e storie di tutto il mondo, ma è stata da alcuni considerata una vera e propria infermità mentale. Una malattia che portava a chi la soffriva ad assumere l'aspetto di un lupo, almeno per un periodo di tempo non troppo lungo, coincidente il più delle volte con il plenilunio. Si veda Gabriele CHIARI, «Il lupo mannaro», in G. Lützenchirchen, Mal di luna, Roma: Newton Compton, 1981, p. 57-81; J.A. MaC CuLlOCH, "Lycanthropy», in J. HASTING (a cura di), Enciclopedy of Religion and Ethics, tomo VIII, p. 206-220; Gaël Milin, Op. cit. e Ermanno PACAGNINI, "Uno sguardo amaro e memoriale», in Il Sole-24 ore, 5 aprile 1992 [ora in Nuove effemeridi, n. 29, 1995, p. 148-149]; H. P. BLum, The Bordeline Childhood of the Wolf-Man, in Freud and his patients, a cura di M. Kanzer e J. Glenn, II, New York-London, 1980, p. 341-58; S. CAMPANELla, Mal di luna e d'altro, Roma: Bonacci, 1986; Gilbert DuRAND, Les structures anthropologiques de l'imaginaire, Paris, Bordas, 1969; M. GARDINER (a cura di), The Wolf-Man by the Wolf-Man, New York, 1971; Carlo GINZBURG, "Freud, l'uomo dei lupi e i lupi mannari», in Id, Miti emblemi spie. Morfologia e storia, Torino: Einaudi, 1992, p. 239-251; F. HAMEL, Human animals, Wellingborough: The Aquarian Press, 1973; Th. LEssing, Haarmann. Storia del lupo mannaro, trad. it. Milano: Adelphi, 1996; Guglielmo LÜTZENKIRCHEN et alt., Mal di luna, con un saggio introduttivo di Alfonso M. Di Nola, prefazione di Nando Agostinelli, Roma: Newton Compton editori, 1981; R. VALENTI PAGNINI, "Lupus in fabula. Trasformazioni narrative di un mito", in Bolletino di studi latini, n. 11, 1981, p. 3-22. Ringrazio l'amico Giosuè Lachin, esperto in luponari, l'arricchimento della mia biblioteca sull'argomento.

21. Carlos Romero (en su edición de Miguel de Cervantes, Los trabajos de Persiles..., cit, p. 244-245) commenta a nota 15 de la p. 244: «[Cervantes] tendría oído y memoria suficiente para recordar un termino siciliano, aunque no privativo, desde luego, «de la mayor isla del Mediterráneo", en la que — no lo olvidemos — vivió bastantes meses y pudo tener efectivo conocimiento de casos de licantropía o manía lupina. En DEI, art. lupomannaro, se lee: «V[oce] di orig. merid., che raggiunge la Sicilia, cfr. abr.[uzzese], lopëmënarë, dal molfett[ano] lëpòmërë, cal[abrese] sett. lëpuómmërë, dal la.t. regionale lupus homines (...) Si error de transcripción sigue habiendo, por parte de C., es ahora mínimo - y comprensible dada la ardua fonética de los dialectos del sur de Italia.» Io penso, invece, che l'etimologia di «luponario» provvenga dal latino lupus hominarius. 
Un urlo bestiale rompeva il silenzio nella notte di luna piena. Ed era uno svegliarsi, un origliare dietro le porte serrate, uno spiare dietro le finestre socchiuse, un porsi in salvo al centro dei crocicchi o impugnare la lama per ferire alla fronte e far sgorgare gocce di nero sangue. Il licantropo s'aggirava per l'abitato, a quattro zampe, ululando, grattando, le porte, con le sue unghie adunche. Il lupo mannaro era l'incubo, lo spavento notturno, nella vecchia cultura contadina, carico di male e malefizio, contro il quale opponeva crudeli gesti esorcistici.

La rappresentazione del licantropo che ulula alla luna all'inizio della narrazione consoliana non lascia indifferenti. La metafora dell'uomo lupo ci parla del dolore straziante di alcuni esseri umani — forse anche dell'intera umanità intesa come singoli individui, famiglie o intere società - contro i quali il destino si è accanito da decenni, se non addirittura da secoli, sotto forma di sfruttamento, depredazione, violenza fisica e morale. Condizione aggravata, nel caso dei Marano e della società siciliana degli anni Venti, dall'avvento del capitalismo selvaggio e soffocante e dall'ascesa del fascismo. La metafora dell'uomo lupo ci parla ancora dell'impossibilità di reagire da parte delle vittime di tale accanimento, se non attraverso la pazzia (di Lucia), la metamorfosi e le urla bestiali (di Marano padre) o il silenzio (del protagonista, Pietro, e di sua sorella Serafina). La licantropia di Marano padre si pone al di là della densità mistica e folclorica della licantropia e del licantropo in generale, già studiata da alcuni autori. C'è infatti in essa una eco di afflizione amorosa che ricorda l'acme dello stato di malinconia d'amore, descritta dal medico catalano Arnau di Vilanova, e da lui denominata cicubus, nel De parte operativa (1271). Tra i sintomi del male non vi è soltanto l'orrore e l'odio irrazionale per la società, ma la convinzione di essere "galli, lupi, cani, vasi di vetro, o di avere la testa di vetro o persino di essere senza testa o anche morti». ${ }^{22}$

Anche il lessico e il ritmo poetico del primo capitolo ci ricordano i poeti lunari che sono, per sua stessa ammissione, i preferiti di Consolo e che non a caso formano con le loro opere la biblioteca ereditata dalla familia Marano e quella privata di Pietro. Penso in particolare a Leopardi (ma riferendoci all'autore, dovremmo citare anche Lucio Piccolo) i cui idilli notturni sono l'ipotesto di alcuni dei momenti di altissima poesia delle descrizioni di questa parte. Basti un esempio: «Nelle solinghe case sopra il colle, scossi nel pietoso sonno del ristoro, credettero ai passi di mali cristiani, ladri o sderegnati che la notte e il vuoto covrono e incorano».

Alla base di questo nucleo narrativo-descrittivo c'è dunque la rappresentazione del malessere dell'umanità o almeno, di quella parte di essa, vittima di soprusi sociali, senza però mai perdere di vista i riferimenti di tipo mitico e archetipico.

22. "Alienatio, quam concomitatur horror, vel odium irrationabile sive immoderatum, et frequenter haec species manifestatur in errore societatis humanae, vel cuiuscumque individui humani (...) Verbi gratia: de melancholicis, utrum aestiment se gallos esse, vel lupos, aut canes, etc. vel utrum esse vitrea vasa, vel caput vitreum, aut non habere caput: et sic de membris aliis: aut se esse mortuos, et non debere comedere.» 
Tenendo ben presente questi due ultimi aspetti, a questo punto dell'analisi emerge prepotentemente la tipologia bestiale di Pan, il grande caprone, figura mitica e archetipica, contrapposta per morfologia e comportamento al luponario che, con le sue zanne e in solitudine, è costretto a vivere il suo calvario lunare. A Pan è dedicata qui di seguito una particolare attenzione.

\section{Janu o il ritorno e la nuova morte di Pan}

"Jeli, lui, non pativa di quelle malinconie», scrive Verga parlando del pastore protagonista della novella omonima. ${ }^{23} \mathrm{E}$ si potrebbe sostituire «Jeli» per «Janu», tale è il collegamento tra il personaggio verghiano e quello di Consolo. Janu, però, è molto di più. Si tratta, innanzi tutto, di uno dei personaggi principali e dei più simbolici della narrazione, visto che le sue vicende occupano gran parte del testo, senza le quali non avremmo diegesi. Egli, come Jeli, è figlio, fratello, amante degli animali con cui vive. Entrambi i protagonisti delle rispettive narrazioni sono anche i pastori che, tra le altre cose, iniziano i giovani figli di famiglia agiata alla conoscenza della natura: Petro (e anche sua sorella Lucia), nel romanzo oggetto di questa analisi. Anzi, l'iniziazione sessuale di Petro, a imitazione dello stesso Janu, avviene con le capre en plein air.

Petro aveva imparato tuttto dalla mandra (...). Avanti Janu, che aveva più di Petro un paio d'anni. E l'aiutò per questo, là alla mandra, a provare una volta con la capra.

«Io la tengo,» disse «sta' sicuro che non guardo... «Meglio che niente, è cosa naturale...»

Una capra lui stesso, Janu («la faccia lunga come quella di una capra»), non proprio incarnazione del dio Pan, ma certo di uno dei suoi fauni.

Janu, inoltre, ha un nome che denuncia l'ambivalenza della sua condotta. Giano è infatti il nome del dio bifronte che annuncia i transiti dal passato al futuro e i cambiamenti da una condizione a un'altra. Janu nel racconto è il protagonista principale del passaggio da un mondo nel quale il mito è vissuto ancora in forma pressocché «naturale», con i suoi riti e rappresentazioni cicliche, a un mondo in cui, invece, il mito è forzato a rinascere imparentato con il diavolo $\mathrm{o}$, meglio detto, trasformato nello stesso Satana. Janu è sì la divinità panica («Piace your divine prick, a real satyr' tool. This sicilian caprone entrare in nostro ovile... Vene, prego, a nostro Tempio, a villa di La pace, all'Abbèi de Thelèm", dice Cronwell, p. 80) che Aleister Crowley — la Grande Bestia 666, to mega therion - cercava negli antenati dell' isola dei satiri, ma anche la vittima propiziatoria del nuovo culto al dio Pan nella sua versione decadente di stregone o diavolo, propria dei costrutti mitici delle contemporane teorie della liberazione. Ma Pan è morto ormai da tempo e il suo ritorno non dipende da

23. Cit. da Giovanni VerGa, Tutte le novelle, a cura di C. Riccardi, Milano: Mondadori, 1979, p. $137-172$. 
queste forme superficiali e decadenti di rievocazione mitica, tutte intrise come sono di erotismo sadomasochista e pertanto non soltanto incapaci di guarire, ma al contrario, buone solo ad ammorbare corpo e anima. Il mito della morte di Pan (Pan, le Grand Pan, est mort) già ascoltato nella tarda antichità, indica che la natura ha finito di parlarci e che tutto è reificato, cessando di essere divinità e diventando semplicemente cosa. ${ }^{24}$ La morte di questo dio che distrugge e insieme preserva, annuncia la scomparsa della società. ${ }^{25}$ Per questo, il povero Janu, oltre a prendersi una malattia venerea, viene accusato di abigeato. Il dio Pan, il caprone divino, è stato degradato a puro pastore, anzi, a mera capra.

Janu è, nella sua ingenuità, un essere ambiguo, senza alcuna malizia, puro istinto ("restò solo nel rifugio della mandra. Privo di pensiero, di volere», p. 71). Nella sua degradazione senza pensiero, Janu coincide pienamente con l'immagine di Pan che il nuovo ordine ideologico insegue per i propri sfoghi falsamente liberatori. Ciò appare chiaramente nella scena dell'orgia panica nella quale Janu, il divine prick (il Pan redivivo), rischia di mancare al suo compito, quando, in piena performance priapica, non riesce a raggiungere l'erezione per penetrare la Cortigiana del Mondo. L'impasse è risolta, insieme alla sensazione di ridicolo che Janu vive, grazie alla cocaina. Tra i partecipanti all'orgia si trova anche il dannunziano Baron Cicio, nemico giurato dei Marano, oltre ad altri seguaci delle diverse manifestazioni dell'irrazionalità dell'epoca. Le quali manifestazioni confluiscono poi nell'ideale fascista e negli ambienti fascisti trovarono anche stimoli rappresentati dalle nuove mode, come l'ostentazione di marche e di marchingegni d'oltralpe (che hanno nella Targa Florio il suo acme), lo snobbismo e la violenza cieca.

Arrivati a questo punto è evidente lo scontro tra due atteggiamenti contrapposti da ogni punto di vista (antropologico, culturale, ideologico, morale e persino mitico) che si concretizza nella descrizione delle due opposte biblioteche (chiaro riferimento alle biblioteche del Fu Mattia Pascal): quella di Mandralisca, cioè del defunto Michele, «zio» di Pietro Marano, e quella del suo avversario, don Nenè Cicio, patrizio di Cefalù. Quest'ultima un coacervo di tradizioni locali, cinismo, volgarità intellettuale e edonismo dannunziano e decadente, quanto la prima è «esercizio ostinato e appassionato della fantasia», fuga della mente verso altre realtà, con la presenza di grandi autori, tra cui brillano in modo particolare Leopardi, Dante, Pascoli, accanto a Tolstoi e Hugo. ${ }^{26}$ Scrittori che alla fine del romanzo Petro contrapporrà anche alla letteratura politica e ai poeti e letterati realisti e dialettali.

\section{James Hillman, Saggio su Pan, Milano: Adelphi, 1977.}

25. "La société tombe en dissolution. Le riche se clôt dans son égoïsme et cache à la clarté du jour le fruit de sa corruption; le serviteur improbe et lâche conspire contre le maître; l'homme de loi, doutant de la justice, n'en comprend ples les maximes; le prêtre n'opère plus de conversions, il se fait séducteur; le prince a pris pour sceptre la clef d'or, et le peuple, l'âme désespérée, l'intelligence assombrie, médite et se tait. Pan est mort, la societé est arrivé au bas» - scrive Proudhon, cit in Jean Chevalier - Alain GHEERBRANT, Dictionaire des symboles, Paris, Robert Laffont, 1969, p. 724.

26. Stefano GIOVANARDI, op. cit., p. 152. 
"Ora - leggiamo in Nottetempo... - sembrava che un terremoto grande avesse creato una frattura, aperto un vallo fra gli uomini e il tempo, la realtà, che una smania, un assillo generale, spingesse ognuno nella sfasatura, nella confusione, nella insania. E corrompeva il linguaggio, strancangiava le parole, il senso loro - il pane si faceva pena, la pasta peste, il miele fiele, la pace pece, il senso sonno...» (p. 140). L'oltraggio e le sue diverse forme, insieme alle svariate reazioni ad esso, è il grande tema di questo romanzo. E l'offesa che ha già colpito le persone (i Marano, Janu) fa scempio anche degli oggetti, come appare chiaramente nel capitolo intitolato "L’oltraggio", in cui degli sconosciuti penetrano in casa Marano per frantumare giare, rovesciare fusti, trafiggere otri. Il desolante spettacolo che lascia dietro di sé la furia devastatrice degli uomini non suggerisce al narratore né amare lamentazioni né veementi denunce contro gli «occulti» mandanti dello scempio, bensì una minuziosa descrizione del processo di realizzazione di un oggetto, una giara, che è stata vittima di tanta furia appunto: l'impasto di creta e acqua che l'artigiano plasma e modella, l'azione solidificatrice del fuoco a cui l'artigiano l'affida, gli asini che la trasportano per i mercati dell'isola e continentali. Dall'evocazione della materialità oltraggiata dell'oggetto scaturisce la spaccatura tra arcaicità e modernità. Tradizioni e geometrie di civiltà soccombono alla violenza cieca dei tempi moderni. In senso più lato è la spaccatura già annunciata simbolicamente dalla morte di Pan che nel romanzo acquista le fattezze di Janu. "Trapassa così l'ignaro pastorello dentro l'irrealtà, viene ridotto a maschera, figura, a bruto strumento di cerimonia» si legge nel capítulo «La calura» (p. 79).

\section{Petro o la malinconia positiva}

E giungiamo finalmente a Petro, protagonista e a tratti voce narrante di Nottetempo.... Anche se la narrazione è a prima vista eterodiegetica, in realtà in molti punti, soprattutto per la focalizzazione costante e per le pause riflessive, risuona la voce di questo personaggio che diventa occhio e penna del narratore, e anche dello stesso autore, quando il romanzo lascia il posto all'autobiografia. Petro attraversa questa storia notturna della Sicilia con quella sua malinconia che lo porta alla fuga dall'isola, deluso dalla politica e dal suo linguaggio, ma destinato a trovare un approdo nella vera letteratura e nella nuova parola.

Consideriamo in primo luogo il nome, Petro. Si tratta della variante semidotta e meridionale del nome latino Petrus, dovuta anche all'influsso del greco ecclesiastico. La pronuncia retroflessa della consonante dentale è un ulteriore marchio di sicilianità. Nella scelta del nome si potrebbe vedere un riferimento al Vangelo di Matteo, là dove Gesù consacra l'Evangelista capo della chiesa cristiana: "Tu sei Pietro, e su questa pietra costruirò la mia chiesa... e ti darò le chiavi del regno dei cieli». Non tralasciando questa metafora della fondazione che sta alla base del nome Petro e comunque al di là di qualsiasi riferimento ecclesiastico, non è da scartare che il nome alluda a un altro tipo di fondazione, quella dell'illuminazione conoscitiva, essa pure associata alla tradizione biblica. 
Petro, però, è qui soprattutto una specie di Sisifo, obbligato a vivere costantemente con la pietra addosso ( 'No, io non sopporto più, più dentro di me questo cotogno», lamentava Petro «sopra di me questo macigno!» e la sua voce sembrava vorticare per le pietre della torre», si legge a p. 37). Lo stesso peso che ha paralizzato Serafina, ha reso pazzo suo padre e sua sorella Lucia. ${ }^{27}$ Un dolore nato molto prima di lui e dei suoi famigliari ( da qualcosa che aveva preceduto la sua, la nascita degli altri.» p. 106), un freddo nell'anima, qualcosa che «era successo al tempo tangeloso dell'infanzia, una rottura, un taglio mai più rimediato» (p. 135), ripete la voce narrante poco prima dell'incontro di Petro con Grazia (non sfugga la grande valenza simbolica anche di questo nome proprio), la donna con cui egli tenta di "frantumare, con furia, senza posa, la pietra del dolore» (p. 135). Petro, dunque, si trova in una situazione opposta a quella dell'apostolo Pietro, perché la sua pietra è dentro di lui e su di essa si ergerà una più profonda conoscenza del mondo: pietra del dolore o della follia che non pietrifica ma che fonda un nuovo linguaggio che non lascia niente d'inesplorato (sentimenti, credenze arcaiche, gesti e volti, movimenti irrazionali e possibilità di redenzione). Forse per questo, all'inizio del capitolo «La torre», Petro che rappresenta la propria dimora come «casa della dolora, patimento, casa dell'innocenza», rifiuta la visione di Dio che gli è sopraggiunta. ${ }^{28}$ Non c'è dubbio che, in questo senso, l'opera è anche un romanzo di iniziazione a un tipo concreto di saggezza, quella letteraria e linguistica che passa, comunque, per la comprensione generale e particolare dell'umano (casa per casa, appunto). Basta leggere, a questo proposito, un frammento del capitolo "Pasqua delle rose»: «Dal piano di essa [la Rocca], da quel cuore partì con il pensiero a ripercorrere ogni strada vicolo cortile, a rivedere ogni chiesa convento palazzo casa, le famiglie dentro, padre figli, i visi loro, rievocarne i nomi, le vicende. Sentiva d'esser legato a quel paese, pieno di vita storia trame segni monumenti. Ma pieno soprattutto, piena la sua gente, della capacità d'intendere e sostenere il vero, d'essere nel cuore del reale, in armonia con esso. Fino a ieri».

Essendo dunque la scrittura e il linguaggio il fulcro del romanzo, è fondamentale seguire con speciale attenzione le tappe attraverso le quali Petro vi accede: dall'impotenza di esprimersi iniziale all'epifania finale. Quasi tutto il capitolo "La torre» gira intorno allo sforzo del protagonista di lasciarsi dietro l'afasia, il grido, l'ululato (anche lui licantropo, in preda al «male catubo»), per appropriarsi finalmente dei nomi («rinominare, ricreare il mondo», p. 39) e con essi del senso, dato che a lui è concesso di non inabbissarsi completamente nella follia, di poter ancora tornare indietro. La luce della candela che compare in queste pagine è metafora della possibilità, non ancora solidamente fondata: sem-

27. E che ha petrificato, se vogliamo, la stessa Sicilia, come Consolo non manca di ripetere ponendo particolare enfasi sulla metáfora della "petrificazione».

28. "No, no!... Non voglio!' e sventagliò le mani per frantumare, fugare quell'immagine, l’immenso dio di tessere che invadeva il cato della fortezza del suo Duomo». Vale a dire il pantocrator del mosaici della cattedrale di Cefalù. 
plicemente «un lume, nella bufera». Questo stesso capitolo registra il tentativo frustrato di accesso alla scrittura:

E nella torre ora, dopo le urla, il pianto, anch'egli, stanco, s'era chetato. Si mise in ginocchio a terra, appoggiò le braccia alla pietra bianca della macina riversa di quello ch'era stato un tempo un mulino a vento, e cercò di scrivere nel suo quaderno - ma intinge la penna nell'inchiostro secco, nel catrame del vetro, nei pori della lava, nei grumi dell'ossidiana, cosparge il foglio di polvere, di cenere, un soffio, e si rivela il nulla, l'assenza d'ogni segno, rivela l'impotenza, l'incapacità di dire, di raccontare la vita, il patimento (p. 53).

A questa straziante dichiarazione d'impotenza segue, alcune pagine più avanti (nel capitolo «Il capretto»), quella della descrizione di una catabasi, una discesa, accompagnata inizialmente dal «chiaror di una lanterna», ${ }^{29}$ dentro «i sotterranei del tempio diruto della Rocca», ma, in realtà, nei luoghi della memoria mitica e materiale dell'isola. Qui ha luogo «un passo nel silenzio», come dice il narratore alla fine di questa scena, vale a dire «un passo all'interno del silenzio e al fine di uscirne». Ë questo un momento centrale: assistiamo a un inabissamento in una "zona incerta" della memoria che recupera assenze, mondi arcaici, miti e età sepolte; negli «spazi inusitati» illuminati da «una luce labile» ma «nuova», grazie alla quale sembra ancora ipotizzabile la narrazione, per quanto fatta di «frasi monche, parole difettive, per accenni, allusioni, per sfasature e afonie». Il narratore, a un certo punto, ricorda, quasi con le parole di Petro, il momento in cui in un passato imprecisato scoprì l'angelo, assorto e fermo, il cui attento sguardo di accecante luce, enuncia enigmi, misteri ed è "preambolo d'ogni altro spettro». Quest'angelo che Petro dice di aver visto nei sotterrani della torre è, in realtà, uno dei bellissimi nunzi dei mosaici del Duomo di Cefalù ${ }^{30}$ come risulta dalla descrizione: «Da sfondi calmi, da quiete lontananze, dagli ocra, dai rosa, dai bruni, da strati sopra strati, chiazze, da scialbature lievi, da squarci in cui traspare l'azzurro tenero o il viola d'antico parasceve». Ma quest'angelo collima con l'altro, lo Scriba, meno esternamente lucente, ma più illuminante internamente, dell'incisione Melencolia I di Dürer: «lo Scriba affaccia, in bianca tunica, virginea come la sua fronte o come il libro poggiato sui ginocchi. (...) È l'ora questa degli scoramenti, delle iner-

29. Ricordiamo, a riprova dell'importanza di quest'immagine del lume, la sua ripresa in una sequenza di chiara ascendeza pascoliana: «Lo guardò Petro allontanarsi [al padre], dietro una finestra, l'altra [Lucia], trapassa, per le stanze un lume, palpita, s'è spento. L'assale l'infinita pena, lo sgomento, si smuove, spande il dolore che ristagna dentro». Inoltre Consolo aveva voluto inserire l'opera di un anonimo Maître de la Chandelle sulla copertina della prima edizione dell'opera. «È un lume che esprime una fede, e questa fede è la letteratura, l'unico antidoto alla pazzia», scrive FRANCHINI 1992: XII.

30. I mosaici si trovano nell'abside e nella crociera. I primi raffigurano Cristo pantocratore a mezzo busto nel catino, la vergine orante fra i quattro arcangeli: Michele, Gabriele, Uriele, Raffaele nel registro inferiore e i dodici apostoli, raggruppati per sei nei due registri inferiori. Nelle vele della crociera, invece, hanno posto le figure dei serafini e dei cherubini, questi ultimi accompagnati dalle figure degli angeli del Signore. 
zie, degli smarrimenti, delle malinconie senza rimedio, l'ora delle geometrie perfette, delle misure inesorabili, la sfera il compasso la clessidra la bilancia.... (...) l'ora della luce bianca, della luce nera, sospesa e infinita.» (p. 65) Lo stesso Consolo ha confermato la fondatezza del rapporto tra il suo angelo e quello dureriano, per quanto alcuni particolari della descrizione non l'avallino; soprattutto il riferimento al volto bianco, poiché è noto che quello dell'angelo della malinconia è nero. ${ }^{31}$ A meno che non si voglia ritenere che alcune di queste divergenze appartengano alle zone d'intersezione tra le due immagini angeliche, se non addirittura a una vera e propria contaminazione fra le due iconografie il cui risultato sarebbe un lettura in positivo, un vero e proprio superamento, di quella dureriana. Il resto della descrizione dello "Scriba» «in bianca tunica» con il «il libro appoggiato sui ginocchi» nell' "ora delle geometrie perfette, delle misure inesorabili, la sfera il compasso la clessidra la bilancia» ci porta, invece, inesorabilmente alla citata acquaforte dell'incisore di Norimberga. Per non parlare della luce crepuscolare di per sé sufficiente elemento di collegamento fra le due immagini.

Un crepuscolo, quello dell'incisione dureriana «magicamente illuminato — detto con parole degli studiosi tedeschi — dal chiarore di fenomeni celesti, che fanno sí che il mare nel fondo brilli di una fosforescenza, mentre il primo piano sembra illuminato da una luna alta nel cielo che proietta ombre profonde». È una «doppia luce» (significato letterale di twilight, crepuscolo) altamente fantastica che domina tutta l'immagine, la quale non intende riprodurre le condizioni naturali di una data ora del giorno, ma alludere piuttosto al misterioso crepuscolo di uno spirito, che non riesce a cacciare i suoi pensieri nella tenebra, né a "portarli alla luce». ${ }^{32}$ Il contenuto concettuale di quest'immagine è doppio, come lo è chiaramente la rappresentazione della scena da parte del narratore consoliano («l'ora della luce bianca, della luce nera, sospesa e infinita»). È doppia anche la natura stessa di Crono o Saturno, dio del Tempo, dominatore dell'età dell'oro e divinità triste e detronizzata, generatore e divoratore contemporaneamente di tutto l'esistente; spirito maligno e dio antico e saggio dei malinconici, esseri divini e bestiali allo stesso tempo. Così come l'ambivalenza del verso di Ungaretti citato qualche volta da Consolo: «la notte nelle vene ti sveglierà».

Tutti questi elementi iconografici dureriani (il libro, la geometria e gli oggetti di misura e calcolo), presenti persino in gran parte della tradizione iconografica della malinconia, ${ }^{33}$ rappresentano oggetti già predisposti alla scrittura, alla strutturazione dello spazio in linee e vettori, al calcolo, ma non ancora utilizzati, poiché l'attenzione del protagonista è previa all'azione, cioè tutta rivolta alle profondità della sua mente. La saggezza dei malinconici è figlia delle profondità. Ma ci vuole l'angelo della luce per passare a uno stadio superiore

31. Si veda KLIBANSKY-PANOFSKY-SAXL, Op. cit., p. 272-273 y 300.

32. KLIBANSKY-PANOFSKY-SAXL, Op. cit., p. 300.

33. Walter Benjamin, Il dramma barocco tedesco, Torino: Einaudi, 1980, p. 145-161. Per quanto riguarda gli oggetti si veda KLIBANSKY-PANOFSKY-SAXL, Op. cit., p. 287-297. 
di linguaggio e conoscenza. Un angelo bianco che era stato annunciato pagine addietro dalla figura vestita di bianco di Lucia, sorella di Petro, quando questi la consegna al ricovero di malati mentali di Bàida (in arabo, bianco) in preda alle allucinazioni («Mi parlano, mi parlano»). ${ }^{34}$

$\grave{E}$ anche indubbio che nella scena narrativa a cui ci siamo dedicati vengono esplorate le rovine di quella Sicilia cantata in diversi luoghi delle Metamorfosi ovidiane e nell'Eneide di Virgilio (soprattutto nel libro III). A essi rimandano gli «abissi, i vuoti, il nulla che s'è aperto ai nostri occhi» dai quali emergono "frammenti, schegge», giacché per un istante il mondo lascia scorgere le sue profondità. Si tratta sicuramente di una scena importante per il suo legame con la malinconia generosa o positiva che è il fondamento psicologico di tutte le arti, la conditio sine qua non di ogni invenzione poetica; un fascio di luce intensissimo che fa risplendere le cose e l'esperienza che di esse abbiamo. ${ }^{35}$ È lo sguardo fisso dell'Angelus novus con gli occhi spalancati, la bocca aperta e le ali dispiegate. "L'angelo della storia - scrive Benjamin nella Tesi IX delle note "Tesi della Filosofia della Storia" 36 — deve avere questo aspetto. Ha il viso rivolto al passato. Là dove davanti a noi appare una catena di avvenimenti, egli vede un'unica catastrofe, che ammassa incessantemente macerie su macerie e le scaraventa ai suoi piedi. Egli vorrebbe ben trattenersi, destare i morti e riconnettere i frantumi. Ma dal paradiso soffia una bufera che si è impigliata nelle sue ali, ed è cosi forte che l'angelo non può più chiuderle. Questa bufera lo spinge inarrestabilmente nel futuro, a cui egli volge le spalle, mentre cresce verso il cielo il cumulo delle macerie davanti a lui. Ciò che chiamiamo progresso, è questa bufera.»

La malinconia è dunque la dimensione dalla quale è possibile comprendere la profondità del mondo; è quindi il tentativo disperato di salvare le cose dal loro precipitare nelle fauci del tempus edax (Che non consumi tu Tempo vora$c e$, ripete il narratore citando Ovidio). Scrive Benjamin in una pagina che amo citare e che qui mi sembra piuttosto pertinente: «La melancolia tradisce il mondo per amore di sapere. Ma la sua permanente meditazione abbraccia le cose morte nella propria contemplazione, per salvarle.»

La fuga di Petro è anche una fuga sia dal silenzio sia dal grido, per aprirsi finalmente alla possibilità di accedere alla parola. Se quella del padre, il luponario, era la fuga nella notte della ragione, nella nera irragionevolezza, nel grido della disperazione, quella di Petro può raggiungere finalmente l'espressione vera che rivela fino in fondo l'angoscia. La Tunisia, in cui approda Petro, è cer-

34. Le riflessioni di Massimo CACCIARI, L'Angelo necessario, Milano: Adelphi, 1986 sull'angelo nuovo hanno molti punti di contatto con questi nostri angeli, in particolar modo il capitolo II ("Angelo e demone»), dove leggiamo: «Il paradiso è perduto per sempre cercato, ma la ricerca si svolge "sin luz para siempre". Essi [gli angeli] inondano il quotidiano, la loro dimora non è più in nessun modo inseparabile dall' aria che l'uomo respira». Si veda anche José JiMÉNEZ, El ángel caído, Barcelona: Seix Barral, 1982.

35. Eugenio Borgna, Op. cit., 1992, P. 149

36. Walter Benjamin, Sul concetto di storia, a cura di Gianfranco Bonola e Michele Ranchetti, Torino: Einaudi, 1997. 
tamente un omaggio dell'autore a una delle prime destinazioni degli espatriati siciliani, ${ }^{37} \mathrm{ma}$ è anche, e soprattutto, la prospettiva dalla quale, liberi dall'oppressione ambientale, si può finalmente guardare e capire la martoriata Sicilia, così come insegnano tanti grandi scrittori siciliani, quali Verga, Capuana, Vittorini che hanno potuto parlare della loro terra solo quando si trovavano lontano da essa.

Il libro finisce come è cominciato, con un plenilunio che illumina il camposanto. Selene, però, non diffonde il suo distaccato candore sui passi disperati di un licantropo, ma è il lume amico che rischiara la strada, non dico verso la guarigione, ma verso un sapere che aiuti a capire il mondo; invece di accompagnare con fredda luce una delle sue creature condannate, ispira un futuro poeta, giacché la conoscenza è fulgore - folgorazione - lunare. Per giungere al punto in cui la luna è sole che illumina il mondo interiore del soggetto, è stato necessario passare attraverso il dolore e la disperazione, conoscere tutte le case e le cose, le pareti, le pietre; leggere tutti i libri, assistere alla degradazione bestiale di amici e nemici, alla degradazione delle tradizioni, dei costumi e dei miti, alla depravazione dei presunti liberatori dello spirito, alla violenza criminale dei fascisti, alla cieca ottusità dei millenaristi politici di qualsiasi specie («Petro costernato aveva visto ancora in quel vecchio la bestia indomita. La bestia dentro l'uomo che si scatena e insorge, trascina nel marasma. La bestia trionfante di quel tremendo tempo, della storia, che partorisce orrori, sofferenze» - p. 170). Petro ha dovuto attraversare il silenzio e il grido: «Ma prima è l'inespresso, l'ermetico assoluto, il poema mai scritto, il verso mai detto. È il sibillino, il mùrmure del vento, frammento, oscuro logo, profezia dei recessi. È la ritrazione, l'afasia, l'impetramento la poesia più vera, è il silenzio. O l'urlo disumano» — si legge a p. 164, giusto prima che le parole e lo stile adeguati escano finalmente dalla penna, si stampino sulla pagina.

Da qui deriva, per conseguenza, la critica sia del realismo che del decadentismo letterari. Un nuovo tipo di letteratura è necessario. Dalla catabasi, dal descensus nella memoria addolorata del mondo siciliano deve sorgere un nuovo stile che riunisca senza distinzioni gerarchiche tutti i livelli di realtà della

37. Come ci ricorda lo stesso Vincenzo CONSOLO in «Il ponte sul canale», ora in Di qua dal faro, p. 219: «Riprende l'emigrazione italiana nel Maghreb nei primi anni dell'Ottocento. E un'emigrazione questa volta intellettuale e borghese, di fuorusciti politici, di professionisti, di imprenditori. Liberali, giacobini e carbonari, si rifugiano in Algeria e in Tunisia [...]. A Tunisi s'era stabilita da tempo una nutrita colonia di imprenditori, commercianti, banchieri [...]. Accanto alla borghesia, v'era poi tutto un proletariato italiano di lavoratori stagionali, pescatori di Palermo, di Trapani, di Lampedusa che soggiornavano per buona parte dell'anno nelle coste maghrebine. // Ma la grossa ondata migratoria di bracciantato italiano in Tunisia avvenne sul finire dell'Ottocento e i primi anni del Novecento, con la crisi economica che colpì le nostre regioni meridionali. [...] Alla Goletta, a Tunisi, in varie città dell'interno, v'erano popolosi quartieri che erano chiamati "Piccola Sicilia" o "Piccola Calabria”.» È una storia troppo complessa, quella delle conseguenze che hanno avuto, in seguito, la guerra di Libia, il fascismo, la seconda guerra mondiale sulle comunità siciliane e italiane di Tunisia per poterla riassumere qua. Lo stesso Consolo rimanda al libro di Nullo Pasotti, Italiani e Italia in Tunisia. 
storia. Non una narrazione lineare, ma una storia piena di tortuosità, a strati sovrapposti, con personaggi, sensazioni e sentimenti del presente, del passato prossimo e di quello più remoto, che attinge a miti a cui si rifanno $i$ comportamenti, in una frantumazione caleidoscopica. Non una lingua standard, ma quella più vicina al punto dal quale sgorgano le emozioni, alla gola strozzata di chi sta per emettere un grido che, invece, si trasforma in una frase, mozza e imperfetta, ma già intelligibile; una lingua piena di terrori e di incertezze di chi si dibatte tra l'impossibilità dell'esprimersi e la necessità di parole, di storia, di narrazione. ${ }^{38}$ La verticalità poetica opposta alla piatta orizzontalità della lingua standard. L'enumerazione, il pastiche, il miscuglio formato da italiano alto (pieno di cultismi, arcaismi, inversioni, iperbati) e una accozzaglia di termini siciliani, spesso impenetrabili per un lettore non isolano, non rispondono al gusto di un mero "edonismo linguistico», di un puro artificio, ma a una scelta linguistica concreta che obbedisce al mondo interiore di questo Figlio del Lupo, il Wölfing. Egli esprime il dolore e la rabbia con il nuovo linguaggio che deriva dalla folgorazione della malinconia positiva in cui ha trasformato la sua pietra del dolore, la sua tristezza abissale. L'identità Petro-narratore è, credo, la conseguenza logica di quello che abbiamo appena detto: la fine del romanzo coincide con il suo stesso inizio, vale a dire che la storia inizia quando la voce narrante può finalmente raccontare il suo travagliato accesso alla scrittura. Nottetempo... è dunque l'autobiografia di Petro Marano, il figlio del bastardo, il capro malinconico che ha attraversato il dolore, la storia notturna sua e del suo paese, la Sicilia. ${ }^{39}$

38. Per una ricerca più approfondita della lingua di Consolo, si veda Salvatore C. TrovaTO, "Forme e funzioni del linguaggio», in Nuove effemeridi, n. 29, 1995, p. 15-29.

39. Invece l'identità Petro - Consolo è più complessa, e in un certo modo meno interessante, per quanto essenziale per capire il valore del linguaggio con il quale entrambi tentano di recuperare Pan e l'irrazionalità dell'istinto, considerati parte della nostra stessa realtà. Non si tratta di un programma di recupero superficiale degli istinti, come quello ideato dal satanista Crowley, ma di riappropriarsi delle caverne di Pan e di Licaone come parte della nostra mente notturna. Se anche essa non potrà ridarci la salute, ci renderà comunque più chiaroveggenti. 